\title{
Construction and Evaluation of a Combined Cyclophosphamide/Nanoparticle Anticancer Vaccine
}

\author{
Kurt Andrew Yaeger, Robert Anthony Kurt
}

Department of Biology, Lafayette College, Easton, USA.

E-mail:kurtr@lafayette.edu

Received June $9^{\text {th }}, 2011$; revised July $8^{\text {th }}, 2011$; accepted July $16^{\text {th }}, 2011$.

\begin{abstract}
Tumor immunotherapy is a rapidly emerging form of cancer treatment. In the current study, a nanoparticle-based vaccine was constructed and the efficacy was assessed through analysis of immune cell populations, tumor growth rates, and metastasis. The vaccine was fabricated through encapsulation of plasmid DNA encoding the tumor-associated antigen Mage-b, and the TLR9 agonist CPG oligodeoxynucleotides by a biodegradable polymer, poly(L,D-lactic-coglycolic acid) (PLGA). The size and shape of the nanoparticles suggested that they were an appropriate size for uptake by professional antigen presenting cells; dendritic cells. Furthermore, effects of the immunopotentiating drug cyclophosphamide was included to decrease systemic populations of regulatory T cells (Treg); immune system sentinels that down-regulate immune responses. The vaccine was assessed using the 4T1 murine mammary carcinoma model which is a model for stage IV breast cancer. The combined cyclophosphamide/nanoparticle vaccine was shown to significantly reduce $4 T 1$ tumor growth rates and lung metastasis in female $B A L B / c$ mice.
\end{abstract}

Keywords: Nanoparticle Vaccine, Breast Cancer, Metastasis, $4 T 1$

\section{Introduction}

In theory, an adaptive immune response could efficiently control a neoplastic growth provided specific tumorassociated antigens (TAA) exists within the malignant cell population to prompt the response [1]. Unlike conventional cancer treatments, this therapeutic modality would be specific and systemic, able to target single cancerous cells as well as distant metastases without cytotoxic side effects on healthy cells [2]. For instance, the TAA Mage- $b$ is a member of the melanoma antigen (MAGE) family of TAA which is overexpressed by many different tumors and exhibits low levels of expression by most normal adult tissues [3]. Indeed vaccination with Mage-b was able to influence growth and metastasis of the aggressive murine mammary carcinoma model $4 \mathrm{~T} 1[4,5]$. However, there are several factors that prevent complete tumor rejection by host immune function. Examples of these factors include: (1) inadequate antigen presentation by immune cells such as macrophages and dendritic cells (DC) [6], (2) poor distinction between TAA and normal self epitopes [7], and (3) the accrual of immunotolerance towards TAA [8]. Recently, methods of overcoming such barriers have been established, paving the way for more effective cancer vaccines.

DC are antigen-presenting cells (APC) that promote stimulation of naïve $\mathrm{T}$ lymphocytes through antigen engulfment and subsequent presentation of the antigen by major histocompatibility complex (MHC) proteins [9]. Upon encountering an antigen, peripheral DC mature and migrate to the nearest lymph nodes, presenting epitopes to $T$ cells to trigger cell mediated immunity [10]. Unfortunately, TAA do not often elicit an immune response sufficient enough for tumor rejection. However, nanoparticles may be able to selectively deliver TAA to DC since peptide or DNA-based antigens encapsulated by PLGA nanoparticles are efficiently taken up by DC $[11,12]$. The spherical shape and size range of these nanoparticles allow for efficient phagocytosis and antigen presentation by DC. Also, due to extended delivery of nanoparticle encapsulated antigens there exists prolonged antigen exposure to $\mathrm{DC}$, a characteristic that generates a more potent immune response.

Although a nanoparticle-based vaccine can be effective, many tumor antigens are indistinct from normal self-proteins and therefore may be passed over by DC 
immunosurveillance [13]. In these situations, toll-like receptor (TLR) activation may be of benefit [14]. TLR detect a wide spectrum of pathogen associated molecular patterns (PAMPs), from unique bacterial products such as lipoproteins (TLR2) and flagellin (TLR5), to nucleic acid motifs intrinsic to bacterial or viral sources (TLR3, 7-9), and initiate expression of inflammatory mediators that regulate inflammation. Moreover, TLR activation of DC promotes the transport of peptide/MHC complexes to the plasma membrane enhancing antigen presentation [15]. This process underscores the vital role TLR play in regulating both local and systemic inflammation, via the activation of DC. Although TLR deal primarily with distinctly microbial antigens, their activation greatly potentiates an immune response allowing TLR agonists to be used as adjuvants. Recently, the activity of TLR agonists in solid tumors has been elucidated. In the presence of TAA, DC activation by the TLR9 agonist CpG oligodeoxynucleotides (CpG ODN) initiates the capture, processing, and presentation of TAA by DC [16]. A TAA vaccine delivered with $\mathrm{CpG}$ ODN as an adjuvant is therefore more immunogenic than the vaccine alone [17]. Similar results were observed in two studies after DC stimulation by nanoparticle encapsulated TAA with TLR3 and TLR4 agonists [18,19]. In both cases, nanoparticle/adjuvant delivery led to DC maturation and migration to the lymph nodes for antigen presentation.

The potency of such vaccines, however, may be hindered by immunotolerance towards TAA. Immunotolerance, a state induced by regulatory $\left(\mathrm{CD} 25^{+} \mathrm{CD} 4^{+} \mathrm{FOXP} 3^{+}\right)$ $\mathrm{T}$ cells (Treg), normally follows a period of infection and is defined by the cessation of an adaptive immune response in order to prevent chronic inflammation [20]. In many cancers, persistent antigen presentation can lead to tolerance, a result of specific Treg cell accumulation for certain TAA [21]. Tumor-associated Tregs can suppresses natural killer (NK) cell function and inhibit activity of APC and T cells through the steric obstruction of MHC molecules, or release of interleukin-10 (IL-10) and transforming growth factor-(TGF- $\beta$ ). While immunotolerance seems to be a natural tendency of the inflammatory process, much progress has been made in artificially arresting Treg cell mediated immune suppression, allowing a persistent, unabated immune response [22]. Cyclophosphamide, originally an anti-tumor chemotherapeutic agent, has been shown on several occasions to inhibit Treg cell activity and potentiate CTL responses when given in combination with a DC vaccine [23-27]. Furthermore, cyclophosphamide treatment of tumor-bearing mice elevates $\mathrm{CD}^{+} / \mathrm{CD}^{+}$, and $\mathrm{CD}^{+} / \mathrm{CD}^{+} \mathrm{T}$ cells within tumors [28]. Thus, priming the tumor microenvironment with cyclophosphamide prior to treatment with a cancer vaccine may enhance anti-tumor immunity.
The present study examined the combined effect of cyclophosphamide treatment and nanoparticle vaccination in tumor-bearing mice. Prior to any immunostimulation, $\mathrm{BALB} / \mathrm{c}$ mice with $4 \mathrm{~T} 1$ tumors were treated with consecutive low doses of cyclophosphamide to deplete Treg cells [26]. Then, using nanoparticles containing a vector encoding Mage-b as well as CpG ODN, as TAA and TLR ligand respectively, mice were vaccinated and subsequently followed for tumor growth and metastasis. The results show that this three-pronged strategy significantly influenced tumor growth and lung metastasis of the aggressive murine mammary carcinoma model $4 \mathrm{~T} 1$. Thus, depleting Tregs, in combination with boosting innate as well as adaptive anti-tumor immunity using a nanoparticle-based vaccine holds promise as a therapeutic vaccine approach.

\section{Material and Methods}

\subsection{Mice and Cell Lines}

4T1 tumor cells used for this study were maintained in complete RPMI (cRPMI) (RPMI 1640, Lonza, Walkersville, MD) supplemented with $10 \%$ heat-inactivated fetal bovine serum (Lonza), glutamine ( $2 \mathrm{mM}$, Lonza), penicillin $(100 \mathrm{U} / \mathrm{mL}$, Lonza), streptomycin (100 ug/mL, Lonza), nonessential amino acids (Sigma, St. Lois, MO), 2-mercaptoethanol $\left(5 \times 10^{-5} \mathrm{M}\right.$, Sigma), and sodium pyruvate $(1 \mathrm{mM}$, Lonza). Balb/c mice were bred on site and were housed in a thoren caging system (Thoren Caging Systems Inc., Hazelton, PA). Food and water were provided ad libitum. All mice were used in accordance with an Institutional Animal Care and Use Committee approved protocol that followed the guide-lines for ethical conduct in care and use of animals.

\subsection{DNA Preparation}

The vector encoding Mage-b for use in the vaccine was generously provided by Dr. Claudia Gravekamp (Department of Cellular and Structural Biology, University of Texas Health Science Center). One Shot Chemically Competent E. coli (Invitrogen, Carlsbad, CA) were transformed with the vector. Bacteria were incubated on ice for 30 minutes and heat shocked for 30 seconds at $42^{\circ} \mathrm{C}$. After immediate transfer to ice, $250 \mu 1$ of room temperature SOC media (Invitrogen) was added. The reaction was shaken horizontally at $200 \mathrm{rpm}$ (Innova 4300, New Brunswick Scientific, Edison NJ) and $37^{\circ} \mathrm{C}$ for 1 hour, plated on Luria-Bertani media containing ampicillin $(50 \mathrm{ug} / \mathrm{ml}, \mathrm{LB} / \mathrm{amp})$ plates and incubated overnight at $37^{\circ} \mathrm{C}$.

Due to the large amount of DNA required for production of the vaccine ( $\sim \mathrm{mg}$ ), purification was carried out using a QIAfilter MAXI filtration kit (QIAGEN, Valen- 
cia, CA). One isolated colony from a LB/amp plate was added to $5 \mathrm{ml} \mathrm{LB} / \mathrm{amp}$ broth and incubated overnight at $37^{\circ} \mathrm{C}$, shaking at $150 \mathrm{rpm}$ (Innova 4300). Next, $200 \mu \mathrm{l}$ of this starter culture was diluted in $100 \mathrm{ml} \mathrm{LB} / \mathrm{amp}$ medium and incubated at $37^{\circ} \mathrm{C}$ for 16 hours. The culture was split equally between four $50 \mathrm{ml}$ tubes and centrifuged at $4^{\circ} \mathrm{C}$ and $6370 \times g$ for 15 minutes. Cell pellets were resuspended in $2.5 \mathrm{ml}$ Buffer P1, and separate tubes were combined. To this, $10 \mathrm{ml}$ Buffer P2 was added, and the solution was incubated at room temperature for 5 minutes. Ten $\mathrm{ml}$ chilled Buffer P3 was added, and after room temperature incubation for 10 minutes, the lysate was passed through a QIAGEN-tip 500 filter, which had been equilibrated by gravity filtration of Buffer QBT. The QIAGEN-tip 500 was washed twice with $30 \mathrm{ml}$ Buffer QC, the DNA was eluted with $15 \mathrm{ml}$ Buffer QF into a $50 \mathrm{ml}$ tube, and precipitated by adding $10.5 \mathrm{ml}$ isopropanol. The reaction was mixed and centrifuged at $5000 \times g$ for 60 minutes. After decanting the supernatant, the DNA pellet was washed with $5 \mathrm{ml} 70 \%$ ethanol and centrifuged at $5000 \times g$ for 60 minutes. The pellet was dried in air and DNA yield was quantified by absorbance at $260 \mathrm{~nm}([\mathrm{DNA}]=\mathrm{A} 260 \mathrm{~nm} \times$ dilution $\times 50 \mathrm{ng} / \mathrm{ul})$ after resuspension in water.

\subsection{Vaccine Preparation}

The PLGA nanoparticle vaccine, loaded with the Mage-b vector and $\mathrm{CpG}$ ODN was prepared by the double emulsion solvent evaporation method [29]. For this purpose the vector encoding Mage-b DNA $(\sim 2.0 \mathrm{mg})$ was diluted in $300 \mu \mathrm{l} \mathrm{CpG} \mathrm{ODN} \mathrm{(Invivogen,} \mathrm{San} \mathrm{Diego,} \mathrm{CA)} \mathrm{solu-}$ tion $(50 \mu \mathrm{g} / \mathrm{ml})$ and $200 \mu 11 \%(\mathrm{w} / \mathrm{v})$ PVA in water. For the first emulsion, $200 \mathrm{mg}$ of the PLGA polymer (Sigma-Aldrich, St. Louis, MO) was dissolved in $2 \mathrm{ml}$ dichloromethane (DCM), and $100 \mu 1$ of the Mage-b/CpG solution was added. Using a microtip probe sonicator (Sonic Dismembrator Model 100, Fischer Scientific, Pittsburgh, PA), the reaction was pulsed at level 2 for 20 seconds. This emulsion was immediately added to 100 $\mathrm{ml} \mathrm{1 \% (w/v)} \mathrm{Polyvinyl} \mathrm{Alcohol} \mathrm{(PVA)} \mathrm{in} \mathrm{water,} \mathrm{forming}$ the second emulsion. The reaction was rapidly stirred overnight at room temperature to evaporate the DCM. The product was washed six times with $50 \mathrm{ml}$ sterile distilled water and frozen overnight at $-80^{\circ} \mathrm{C}$. Nanoparticles were then lyophilized and stored until use in a desiccator at room temperature.

\subsection{Nanoparticle Analysis}

A particle size distribution was obtained using a Zetasizer (Zetasizer Nano, Malvern Instruments Ltd, Worchestershire, UK). The procedure assesses the Brownian motion of a sample of particles, and correlates this random diffusion to particle radius via the Stokes-Einstein equation. The Zetasizer detects dynamic light scattering of a population of particles, which is a function of diffusion. Nanoparticles were visualized by scanning electron microscopy (Department of Colloids, Max Planck Institute for Colloids and Interfaces). A release assay was performed to quantify the hydrolysis and release of DNA from the nanoparticles with respect to time. For this purpose, nanoparticles were resuspended in $5 \mathrm{ml}$ sterile distilled water and incubated with shaking at $37^{\circ} \mathrm{C}$ for fifteen days. Three samples were taken each day and analyzed for absorbance at $260 \mathrm{~nm}$ to quantify DNA concentration.

\subsection{Vaccination and Tumor Growth}

For each experiment 30 mice received $5 \times 10^{4} 4 \mathrm{~T} 1$ tumor cells in $100 \mu \mathrm{l}$ HBSS. Ten mice in each group were maintained as a positive control. The 20 remaining individuals were injected intraperitoneally with $20 \mathrm{mg} / \mathrm{kg}$ cyclophosphamide 4,3 , and 2 days prior to nanoparticle vaccination. The cyclophosphamide protocol was developed by Barbon et al. [26] and shown to effectively decrease Treg cell activity. Ten of the cyclophosphamide-treated mice received $100 \mu \mathrm{l}$ of the nanoparticle vaccine in HBSS $(3 \mathrm{mg} / \mathrm{ml})$, injected into the left tibialis muscle. Figure 1 summarizes the timeline for treatment of the mice. Tumor growth rates were determined beginning 7 days after nanoparticle delivery using vernier calipers to measure tumor dimensions and calculating tumor volume $=\mathrm{L} \times \mathrm{W}^{2} / 2$.

\subsection{Analysis of Metastasis}

Following sacrifice, lungs were harvested from each mouse to analyze metastases. The tissues were minced and digested in enzyme cocktails containing $1 \mathrm{mg} / \mathrm{mL}$ collagenase type IV (Worthintgon Biochemical Corp., Lakewood, NJ) and $0.1 \mathrm{mg} / \mathrm{mL}$ elastase (Worthington) at room temperature in spinner flasks for 1 hour. The cells were then washed and resuspended in $10 \mathrm{ml}$ cRPMI. Two dilutions per organ were made (9/10 and 1/10) and

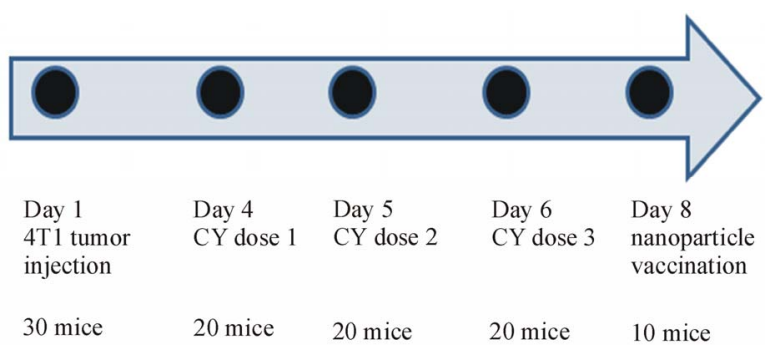

Figure 1. Treatment regimen. For each experiment 30 mice were given tumors on day one. Twenty of the mice received cyclophosphamide (CY) treatment on days 4,5 , and 6 . Ten of the CY treated mice received the nanoparticle vaccine on day 8 . The experiment was completed three separate times. 
plated on tissue culture dishes with $10 \mu \mathrm{M}$ thioguanine (Sigma). Samples were incubated at $37^{\circ} \mathrm{C}$ and $5 \% \mathrm{CO}_{2}$. Fourteen days later the cells were fixed with methanol (Fisher), stained with $0.03 \%$ methylene blue (Sigma), and colonies counted.

\subsection{Flow Cytometry}

Vaccine draining (inguinal) lymph nodes and splenocytes were harvested eight days after vaccination and prepared for flow cytometry. Lymphocytes were removed from the organs by pressing the organs with the flat end of a syringe plunger. For staining, $1 \mathrm{ml}$ of cells at $5 \times 10^{5}$ cells/ml in cRPMI was added to $15 \mathrm{ml}$ tubes and $1 \mu \mathrm{g}$ of each antibody was added. To trace the profile of helper $\mathrm{T}$ cells, cytotoxic $\mathrm{T}$ cells, and regulatory $\mathrm{T}$ cells antibodies specific for CD3, CD4, CD8, CD25, and isotype controls were used (BD Biosciences, San Jose, CA). After incubation on ice for 30 minutes, cells were resuspended in 1 $\mathrm{ml} 3.7 \%$ formaldehyde, incubated again on ice for 10 minutes, and washed with $10 \mathrm{ml}$ HBSS. Cells were resuspended in $1 \mathrm{ml}$ HBSS and sent to Pennsylvania State University Hershey (Hershey, PA) for analysis.

\section{Results}

\subsection{Nanoparticle Analysis}

Before vaccinating mice with the nanoparticles we wanted to determine whether the particles were the desired size and whether the encapsulated DNA would be released in an aqueous environment. Scanning electron microscopy (SEM) images indicated consistencies in shape of the nanoparticles (Figure 2(a)), while a size distribution obtained using a zetasizer revealed the nanoparticles ranged in diameter from approximately $50 \mathrm{~nm}$ to $900 \mathrm{~nm}$, and the distribution centered at approximately $350 \mathrm{~nm}$ (Figure 2(b)). To assess release of the encapsulated DNA the nanoparticles were resuspended in water and samples were taken every 24 hours to assess DNA concentration. The DNA release began within 24 hours and plateaued after 12 days (Figure 3). These data revealed the successful generation of nanoparticles the proper size for DC uptake, and that the nanoparticles were capable of releasing the encapsulated DNA.

\section{2. $\mathrm{T}$ Cell Subsets Are Not Altered by Nanoparticle Vaccination}

In an attempt to gauge whether the vaccine caused an expansion of effector cells we assessed vaccine draining lymph nodes and splenocytes 8 days after vaccination. Within the lymph nodes $\mathrm{CD}^{+} / \mathrm{CD}^{+}\left(\mathrm{T}_{\mathrm{H}}\right), \mathrm{CD}^{+} / \mathrm{CD}^{+}$ (CTL), and $\mathrm{CD}^{+} / \mathrm{CD} 25^{+}$(Treg) cells made up approximately $50 \%, 20 \%$, and $3 \%$ of the cells respectively for all experimental groups (Figure 4). Although the lack of an increase in $\mathrm{T}_{\mathrm{H}}$ or CTL in the lymph nodes was surprising since 8 days following vaccination there should be anongoing immune response relative to control mice, it was not surprising that the Treg cell numbers were normal since the cells were assessed 10 days following the last cyclophosphamide treatment. Analysis of splenocytes
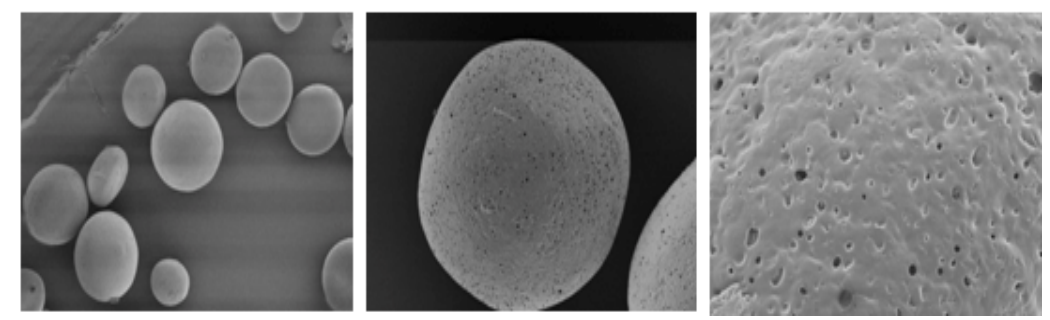

(a)

Size Distribution by Intensity

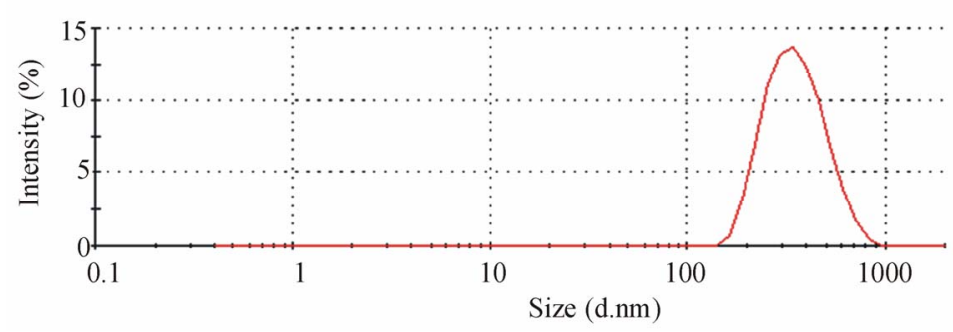

(b)

Figure 2. Nanoparticle shape and size. (a) Scanning electron micrographs of the PLGA nanoparticle vaccine. (b) Nanoparticle size distribution as determined by Zetasizer. The distribution was centered at approximately 350nm. The experiment was run three times, one of which yielded this representative distribution. The size $(d=$ diameter $)$ is measured in nanometers. 


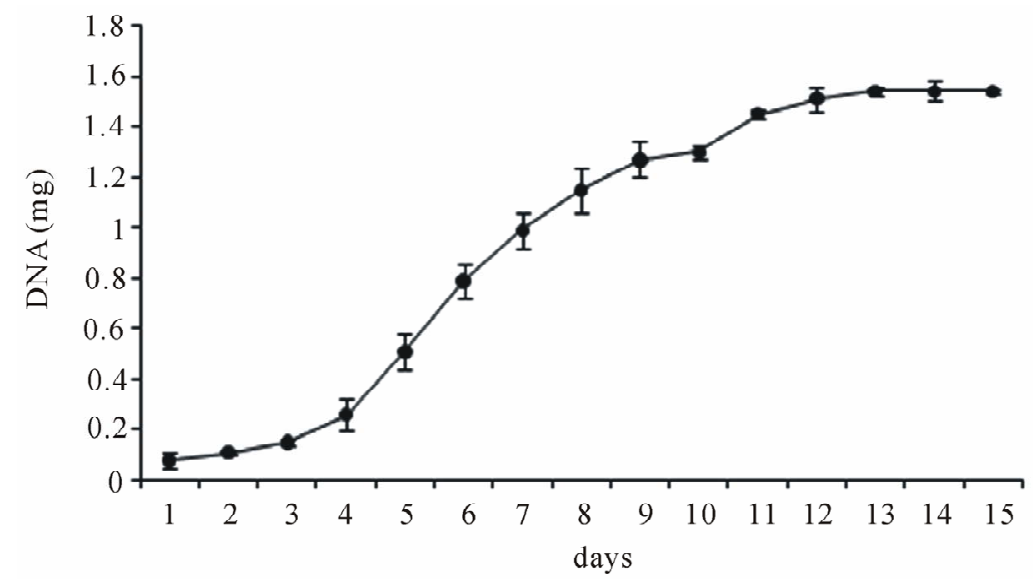

Figure 3. DNA release from the nanoparticles over time. A sample of the nanoparticle vaccine was resuspended in water and maintained at $37^{\circ} \mathrm{C}$ in a shaking incubator. The amount of DNA in solution plateaued after 12 days, indicating complete nanoparticle hydrolysis. The plot contains the average $+/-$ standard deviation of the DNA concentration of the three separate samples taken each day.

also revealed no significant differences between treatment groups. Although there were fewer $\mathrm{T}_{\mathrm{H}}, \mathrm{CTL}$ and Treg cells in the spleens from cyclophosphamide treated and nanoparticle vaccinated mice the differences were not significant (Figure 4). It is possible that the small decrease in these populations could be attributed to cells exiting the spleen and localizing to the tumor site. Regardless, collectively these data reveal that 8 days following vaccination with the nanoparticles there was no obvious expansion of effector $\mathrm{T}$ cells in the spleens or vaccine draining lymph nodes.

\subsection{Tumor Growth and Metastasis Are Decreased by Nanoparticle Vaccination}

To evaluate whether the nanoparticle vaccine influenced tumor progression we monitored tumor growth over time as well as lung metastasis. All tumors, regardless of treatment, followed a relatively exponential growth rate (Figure 5). In the early stages, before the $7^{\text {th }}$ day post-vaccination, tumors in control mice grew faster than tumors in mice treated only with cyclophosphamide and tumors in nanoparticle vaccinated mice. After this point, however, tumors in the cyclophosphamide only treated group began to grow at a rate similar to the controls, whereas mice that received the nanoparticle vaccine continued to exhibit a slower growth rate. Although mice treated with cyclophosphamide alone and mice that received the nanoparticle vaccine both exhibited significantly slower tumor growth rates than control mice, the tumors were much smaller in the mice that received the nanoparticle vaccine. Following 24 days of analysis the tumors in control mice averaged $2753+/-386 \mathrm{~mm}^{3}$, whereas tumors in the cyclophosphamide only treated mice averaged $2213+/-216 \mathrm{~mm}^{3}$, and tumors in the mice that received cyclophosphamide and the nanoparticle vaccine averaged $1107+/-161 \mathrm{~mm}^{3}$ (Figure 5). These data suggest that cyclophosphamide alone has a benefit in leading to a reduction in tumor growth rate, although this reduction was not as significant as when the mice also received the nanoparticle vaccine.

We chose the 4T1 murine mammary carcinoma model for this study because of its aggressive nature and ability to spontaneously metastasize. Since metastasis is often a major contributor to death of patients with cancer we wanted to know whether the nanoparticle vaccine also had any effect on lung metastasis. For this reason, the metastatic ability of primary $4 \mathrm{~T} 1$ tumors from the different treatment groups was assessed by quantifying colonies that grew from the resected and digested lungs. Lung tissue from control mice exhibited the greatest number of metastatic colonies (2306), while cyclophosphamide only treated mice exhibited significantly fewer colonies (1777), and lungs from mice that received the nanoparticle vaccine exhibited the fewest metastatic colonies (1528) (Figure 6). These data suggest that cyclophosphamide alone has a benefit in reducing metastasis, although this benefit was not as significant as when the mice also received the nanoparticle vaccine. Thus, mice that received cyclophosphamide and the nanoparticle vaccine exhibited the greatest reduction in tumor growth rate and lung metastasis.

\section{Discussion}

The size distribution of the nanoparticles, which centered around $350 \mathrm{~nm}$, and spherical shape assortment, indicated by zetasizer analysis and SEM imaging respectively, revealed a nanoparticle vaccine that should be phagocytosed by APC; an advantage of the nanoparticle approach. 


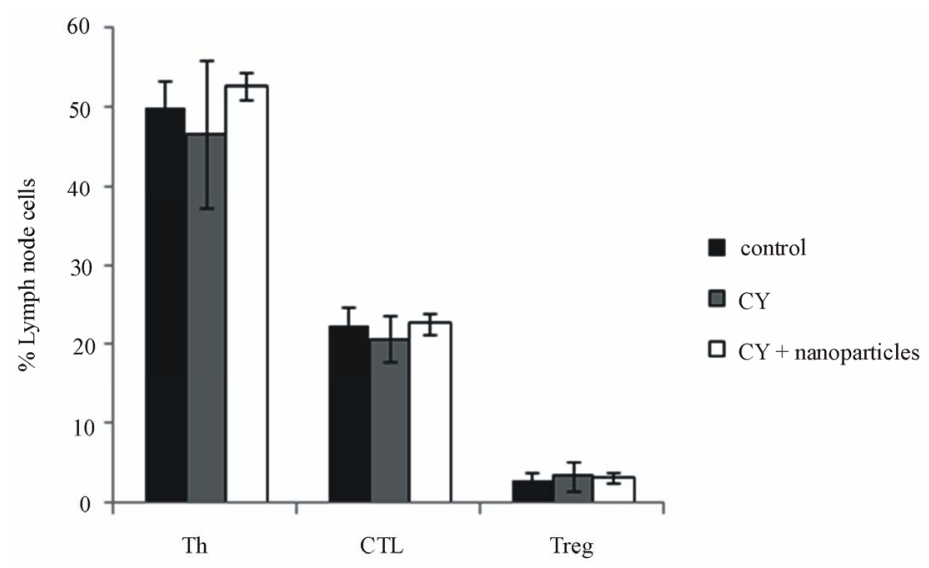

(a)

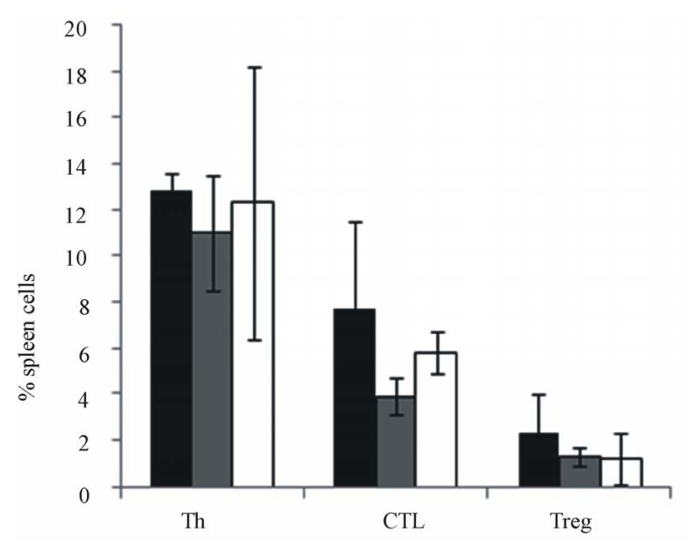

(b)

Figure 4. Analysis of lymphocytes from lymph nodes and spleens. The number of helper T cells (Th), cytotoxic T cells (CTL), and regulatory $T$ cells (Treg) in inguinal lymph nodes (a) and spleens (b) were assessed 8 days after vaccination by flow cytometry. The data represent the average $+/-$ standard deviation of all three experiments.

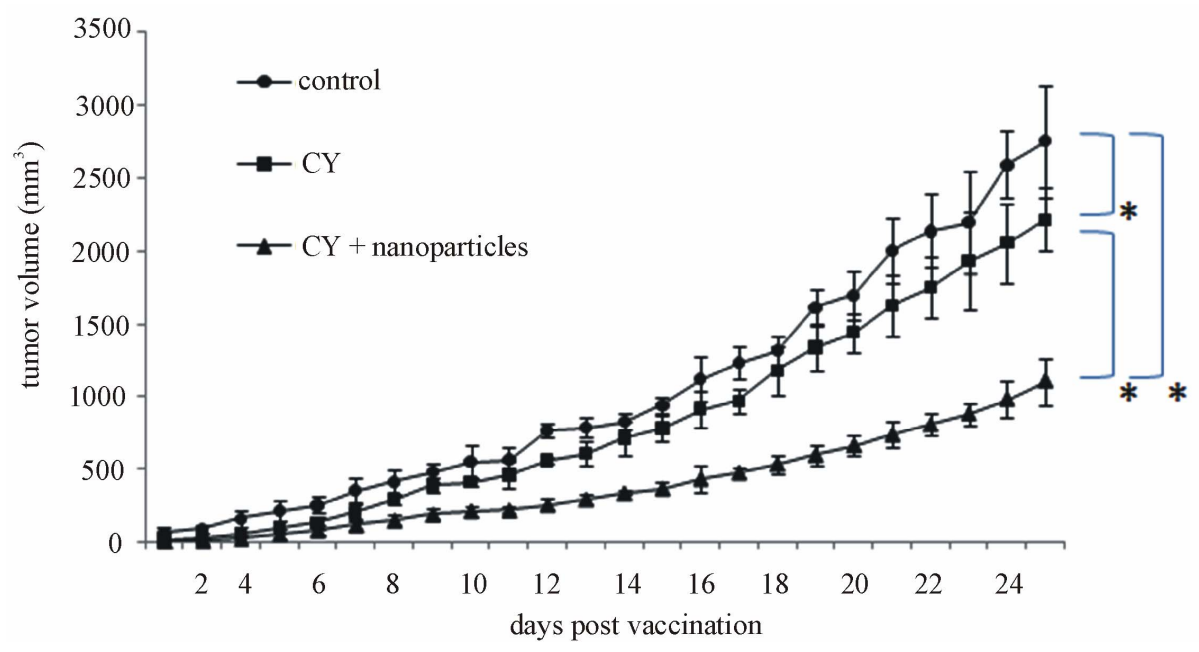

Figure 5. Tumor growth rates. Growth rates of 4T1 tumors from untreated mice (control), mice treated with cyclophosphamide alone (CY), and mice treated with $C Y$ and the nanoparticle vaccine (CY + nanoparticles) are shown. Tumors were measured daily using vernier calipers. The data represent the average $+/$-standard deviation of all three experiments. Where indicated (*) $p<0.001$ using Student's $t$-Test relative to the control. 


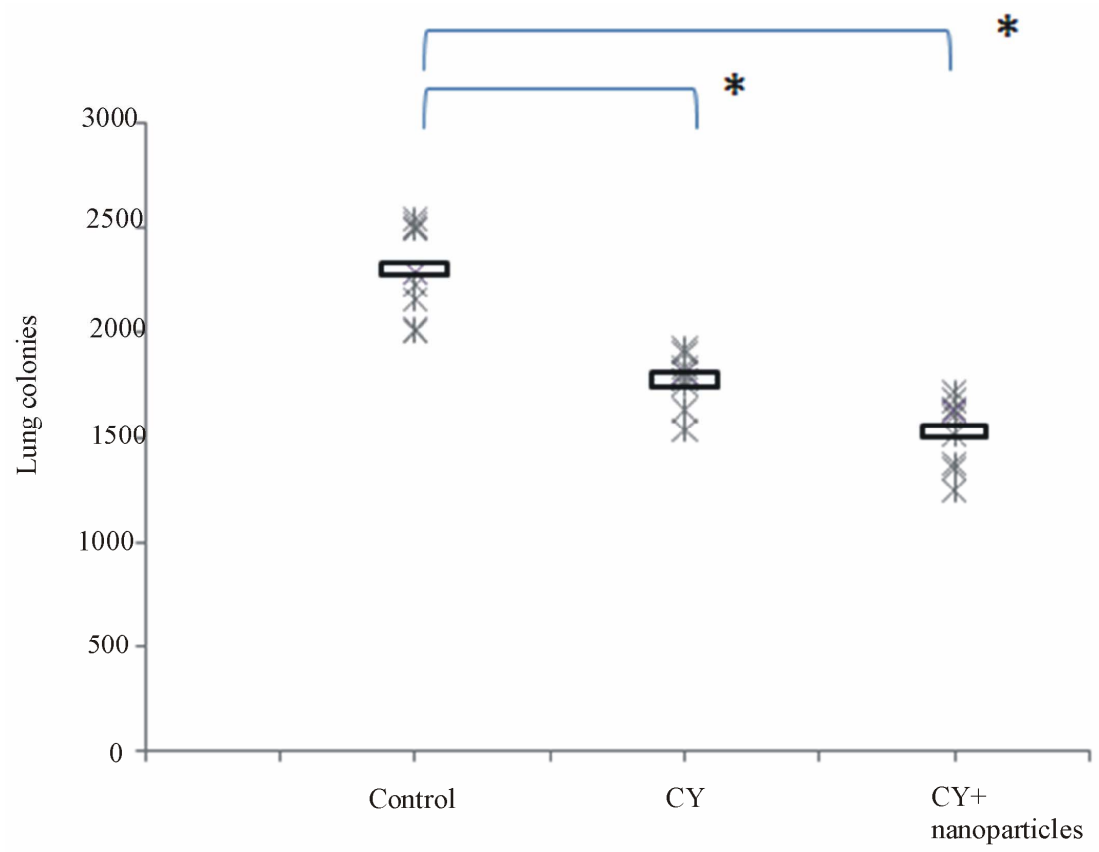

Figure 6. Number of metastatic colonies recovered from the lungs of tumor-bearing mice. Lungs were harvested 24 days after vaccination, processed, and incubated for 14 days before assessing colony outgrowth. The data represent the number of metastatic colonies from each individual mouse (x), and the average ( $\square$ ) of all mice from two separate experiments. Where indicated $(*) p<0.001$ using Student's $t$-Test relative to the control.

For nanoparticles with cytotoxic drugs internalized, this would also limit systemic cytotoxicity. The nanoparticle itself is biodegradable and nontoxic, deteriorating rapidly in solution to an assortment of water-soluble substances, such as lactic and glycolic acids, which are simply excreted [30]. Analysis of release from the nanoparticles indicated a rapid hydrolysis of the particles with DNA release between 4 and 10 days, and complete discharge of all encapsulated DNA after the $12^{\text {th }}$ day. The steady release of antigen over a period of a few days is especially important in eliciting a potent immune response, as it helps maintain antigen concentration.

In theory, there are two mechanisms by which the nanoparticles deliver antigen: through extracellular nanoparticle hydrolysis and antigen release, leading to free antigen uptake by APCs, or through endocytosis of nanoparticles and intracellular antigen release. Although we have not explored which mechanism predominates in this study, elements of both may occur in vivo. Nonetheless, intracellular antigen release is likely an efficient method, and steps could be taken in future studies to emphasize this route. For instance, recent studies have indicated that covalent modifications to the surface of nanoparticles are possible, and indeed allow greater specificity for accumulation and endocytosis $[12,19]$. Thus, modifying the surface of the nanoparticles with antibodies or TLR ligands may increase effectiveness of the vaccine.
In this study, at early stages of tumor growth (1 to 5 days post-vaccination), tumors of the two groups treated with cyclophosphamide had similar growth rates. Initially, cyclophosphamide action would have decreased Treg cells, leading to an increased capacity for an immune response. However, by day 8 , when lymph nodes and spleens were harvested, the effects of the cyclophosphamide treatment seem to have passed as suggested by a similar number of Treg cells in the different experimental groups. These data are in agreement with Barbon et al. [26], who determined that the impact of cyclophosphamide lasts between 6 to 8 days. Around this same time growth rates of tumors in mice treated only with cyclophosphamide began to more closely parallel growth rates in control mice. This may have been caused by tumor-induced immunosuppression as a result of an up-regulation of Treg cells, which inhibits a tumor-specific $\mathrm{T}$ cell response [31].

However, when the nanoparticle vaccine was used in conjunction with cyclophosphamide treatment tumor growth rates were significantly lower throughout the experiment. This may be attributed to a primed immune environment formed through initial cyclophosphamide depletion of Treg cells and followed by an efficient $\mathrm{T}$ cell response initiated by uptake of the nanoparticle vaccine containing DNA encoding the Mage-b TAA to stimulate an immune response, and CpG ODN to stimulate innate immunity. In theory, this anti-tumor immune 
response could have been sustained longer, but in the absence of booster vaccinations, this response was eventually restricted. In many instances, vaccine boosters have been used to maintain the anti-tumor immune response $[10,18,19]$. Although beyond the scope of the current study, it would be interesting to determine whether additional cyclophosphamide treatments and nanoparticle vaccinations could further impact the tumor growth rate. It would also be worthwhile to explore the impact of the nanoparticle vaccine without cyclophosphamide treatment in order to determine the extent to which Treg depletion was important for efficacy of the vaccine. Another interesting area to investigate would be to look at prophylactic vaccination with the vaccine rather than therapeutic vaccination as studied here. With many tumor models prophylactic vaccination often results in a more significant impact on tumor progression than therapeutic vaccination.

Interestingly, we did not find evidence of $\mathrm{T}$ cell expansion following vaccination, and we did not verify whether an antigen-specific immune response was elicited by the vaccine. Subsequent studies are undoubtedly necessary and warranted to delineate how the vaccine works. For this purpose we are interested in looking at further subsets of T cells (Th1, Th2, memory cells, antigen specific tetramer positive cells), as well as additional lymphocyte populations such as B cells, NK cells and $\gamma \delta \mathrm{TCR}+\mathrm{T}$ cells. Initial studies in nude or SCID mice would help delineate whether an antigen specific immune response is important for vaccine efficacy and subsequent studies such as ELISPOT, cytokine release, cytotoxicity, and proliferation assays would be extremely useful in determining how the vaccine works to decrease tumor growth and metastasis.

In this study, the extent of lung metastasis also correlated with tumor growth inhibition. The lung metastasis in mice treated with cyclophosphamide alone and mice treated with the nanoparticle vaccine were significantly less than the lung metastasis in control mice. However, although there were fewer lung metastasis in nanoparticle vaccinated mice, lung metastasis in mice treated only with cyclophosphamide, and mice treated with cyclophosphamide in combination with the nanoparticle vaccine were not significantly different. These data underscore the importance of Treg cell depletion in limiting metastatic growth, and that coupled with a nanoparticle vaccine the effect is even greater. As with tumor growth, it would be interesting to look at the effect of additional cyclophosphamide treatments and booster vaccinations on lung metastasis in future studies.

Collectively, the data presented here indicate a combined cyclophosphamide/nanoparticle vaccine was successful in both significantly slowing primary tumor growth rates and lung metastasis in mice with 4T1 tumors. The data suggest that through a multi-stage mechanism of action, the immune response is augmented upon depletion of Treg cells with cyclophosphamide, and is triggered to respond to the tumor cells with the nanoparticle vaccine containing DNA encoding a TAA and $\mathrm{CpG}$ ODN to stimulate innate immunity. However, inhibition of tumor growth was only temporary; without additional vaccine boosters, the tumors began to grow at an unrestricted rate. The effectiveness of booster vaccinations and additional cyclophosphamide treatments should be evaluated in future studies. In theory, continued treatments could prolong a more extensive anti-tumor immune response. Nonetheless, the results obtained using a therapeutic vaccine approach for this highly aggressive murine mammary carcinoma model highlights the potential for nanoparticle vaccines in eliciting antitumor immunity.

\section{Acknowledgements}

We would like to thank the Department of Biology at Lafayette College for support of this work. We would also like to thank Drs. James Ferri (Department of Chemical and Biomolecular Engineering), David Husic (Department of Chemistry), and Charles Nutaitis (Department of Chemistry) for all of their advice and assistance with the generation of the nanoparticle vaccine.

\section{REFERENCES}

[1] T. Waldmann, "Immunotherapy: Past, Present and Future," Nature Medicine, Vol. 9, 2003, pp. 269-277. doi:10.1038/nm0303-269

[2] J. Blattman and P. Greenberg, "Cancer Immune Therapy: A Treatment for the Masses," Science, Vol. 305, No. 5681, 2004, pp. 200-205. doi:10.1126/science.1100369

[3] C. Lurquin, C. De Smet, F. Brasseur, F. Muscatelli, V. Martelange, E. De Plaen, R. Brasseur, A. P. Monaco and T. Boon, "Two Members of the Human MAGEB Gene Family Located in Xp21.3 Are Expressed in Tumors of Various Histological Origins," Genomics, Vol. 46, No. 3, 1997, pp. 397-408. doi:10.1006/geno.1997.5052

[4] R. K. Sypniewska, L. Hoflack, M. Tarango, S. Gauntt, B. Z. Leal, R. L. Reddick and C. Gravekamp, "Prevention of Metastasis with a Mage-b DNA Vaccine in a Mouse Breast Tumor Model: Potential for Breast Cancer Therapy," Breast Cancer Research and Treatment, Vol. 91, No. 1, 2005, pp. 19-28. doi:10.1007/s10549-004-6454-7

[5] C. Gravekamp, B. Leal, A. Denny, R. Baher, S. Lampkin, F. Castro, S. H. Kim, D. Moore and R. Riddick, "In vivo Responses to Vaccination with Mage-b, GM-CSF and Thioglycollate in a Highly Metastatic Mouse Breast Tumor Model, 4T1," Cancer Immunology Immunotherapy, Vol. 57, No. 7, 2008, pp. 1067-1077. doi:10.1007/s00262-007-0438-5 
[6] J. Banchereau and A. Palucka, "Dendritic Cells as Therapeutic Vaccines against Cancer," Nature Reviews Immunology, Vol. 5, 2005, pp. 296-306. doi:10.1038/nri1592

[7] S. Rakoff-Nahoum and R. Medzhitov, "Toll-Like Receptors and Cancer," Nature Reviews Cancer, Vol. 9, 2009, pp. 57-63. doi:10.1038/nrc2541

[8] M. Colombo and S. Piconese, "Regulatory T-Cell Inhibition versus Depletion: The Right Choice in Cancer Immunotherapy," Nature Reviews Cancer, Vol. 7, 2007, pp. 880-887. doi: $10.1038 / \mathrm{nrc} 2250$

[9] M. Lutsiak, D. Robinson, C. Coester, G. Kwon and J. Samuel, "Analysis of Poly(D,L-lactic-co-Glycolic Acid) Nanosphere Uptake by Human Dendritic Cells and Macrophages in Vivo," Pharmacological Research, Vol. 19, No. 10, 2002, pp. 1480-1487. doi:10.1023/A:1020452531828

[10] T. Yoshikawa, N. Okada, A. Oda, K. Matsuo, K. Matsuo, H. Kayamuro, Y. Ishii, T. Yoshinaga, T. Agaki, M. Akashi and S. Nakagawa, "Nanoparticles Built by Self- Assembly of Amphiphilic Gamma-PGA Can Deliver Antigens to Antigen-Presenting Cells with High Efficiency: A New Tumor-Vaccine Carrier for Eliciting Effector $T$ Cells," Vaccine, Vol. 26, No. 10, 2008, pp. 1303-1313. doi:10.1016/j.vaccine.2007.12.037

[11] Y. Men and M. Groettrup, "PLGA Microspheres for Improved Antigen Delivery To Dendritic Cells as Cellular Vaccines," Advanced Drug Delivery Reviews, Vol. 57, No. 3, 2005, pp. 475-482. doi:10.1016/j.addr.2004.09.007

[12] P. Kocbek, N. Obermajer, M. Cegnar, J. Kos and J. Kristl, "Targeting Cancer Cells Using PLGA Nanoparticles Surface Modified with Monoclonal Antibody," Journal of Controlled Release, Vol. 120, No. 1-2, 2007, pp. 18-26. doi:10.1016/j.jconrel.2007.03.012

[13] M. Diwan, P. Elamanchili, H. Lane, A. Gainer and J. Samuel, "Biodegradable Nanoparticle Mediated Delivery to Human Cord Blood Derived Dendritic Cells for Induction of Primary T Cell Responses," Journal of Drug Targeting, Vol. 11, No. 8-10, 2003, pp. 495-507. doi:10.1080/10611860410001670026

[14] A. Iwasaki and R. Medzhitov, "Toll-Like Receptor Control of the Adaptive Immune Responses," Nature Immunology, Vol. 5, 2004, pp. 987-995. doi:10.1038/ni1112

[15] J. Blander and R. Medzhitov, "Toll-Dependent Selection of Microbial Antigens for Presentation Bydendritic Cells," Nature, Vol. 440, 2006, pp. 808-812. doi:10.1038/nature 04596

[16] A. Krieg, "Toll-Like Receptor 9 (TLR9) Agonists in the Treatment of Cancer," Oncogene, Vol. 27, 2008, pp. 161-167. doi:10.1038/sj.onc.1210911

[17] M. Diwan, P. Elamanchili, M. Cao and J. Samuel, "Dose Sparing of CpG Oligodeoxynucleotide Vaccine Adjuvants by Nanoparticle Delivery," Current Drug Delivery, Vol. 1, No. 4, 2004, pp. 405-412. doi:10.2174/1567201043334597

[18] S. Hamdy, O. Molavi, Z. Ma, A. Haddadi, A. Al-shamsan, Z. Gobti, S. Elhasi, J. Samuel and A. Lavasanifar, "Co-Delivery of Cancer-Associated Antigen and TollLike Receptor 4 Ligand in PLGA Nanoparticles Induces
Potent $\mathrm{CD}^{+}$T Cell-Mediated Anti-Tumor Immunity,' Vaccine, Vol. 26, No. 39, 2008, pp. 5046-5057. doi:10.1016/j.vaccine.2008.07.035

[19] C. Wischke, J. Zimmermann, B. Wessinger, A. Schendler, H. Borchart, J. Peters, T. Nesselhut and D. Lorenzen, "Poly(I:C) Coated PLGA Microparticles Induce Dendritic Cell Maturation," International Journal Pharmacology, Vol. 365, 2009, pp. 61-68.

[20] K. Wing and S. Sakaguchi, "Regulatory T Cells Exert Checks and Balances on Self-Tolerance and Autoimmunity," Nature Immunology, Vol. 11, No. 1, 2010, pp. 7-13. doi:10.1038/ni.1818

[21] W. Zou, "Regulatory T Cells, Tumor Immunity and Immunotherapy," Nature Reviews Immunology, Vol. 6, 2006, pp. 295-230. doi:10.1038/nri1806

[22] R. Schabowsky, S. Madireddi, R. Sharma, E. Yolcu and H. Shirwan, "Targeting $\mathrm{CD} 25^{+} \mathrm{CD} 4{ }^{+} \mathrm{FOXP}^{+}$Regulatory T Cells for the Augmentation or Cancer Immunotherapy," Current Opinion in Investigational Drugs, Vol. 8, 2007, pp. 1002-1008.

[23] J. Taieb, N. Chaput, N. Schartz, S. Roux, S. Novault, C. Menard, F. Ghiringhelli, M. Terme, A. Carperntier, G. Darrasse-Jese, F. Lemonnier and L. Zitvogel, "Chemoimmunotherapy of Tumors: Cyc-Lophosphamide Synergizes with Exosome Based Vaccines," Journal Immunology, Vol. 176, 2006, pp. 2722-2729.

[24] J. Liu, Y. Wu, X. Zhang, J. Yang, H. Li, Y. Mao, Y. Wang, X. Cheng, Y. Li, J. Xia, M. Masucci and Y. Zeng, Single Administration of Low Dose Cyclo-Phophamide Augments the Antitumor Effect of Dendritic Cell Vaccine," Cancer Immunology Immunotherapy, Vol. 56, No. 10, 2007, pp. 1597-1604. doi:10.1007/s00262-007-0305-4

[25] M. Salem, A. Kadima, S. El-Naggar, M. Rubinstein, Y. Chen, W. Gillanders and D. Cole, "Defining the Ability of Cyclophosphamide Preconditioning to Enhance the Antigen-Specific $\mathrm{CD}^{+}{ }^{+} \mathrm{T}$ Cell Response to Peptide Vaccination: Creation of a Beneficial Host Microenvironment Involving Type I IFNs and Myeloid Cells," Journal of Immunotherapy, Vol. 30, No. 1, 2007, pp. 40-53. doi:10.1097/01.cji.0000211311.28739.e3

[26] C. Barbon, M. Yang, G. Wands, R. Ramesh, B. Slusher, M. Hedley and T. Luby, "Consecutive Low Doses of Cyclophosphamide Preferentially Target Tregs and Potentiate T Cell Responses Induced by DNA PLG Microparticle Immunization," Cellular Immunology, Vol. 262, No. 2, 2010, pp. 150-161. doi:10.1016/j.cellimm.2010.02.007

[27] V. Radojcic, K. Bezak, M. Skarica, M. Pletneva, K. Yoshimura, R. Schulick and L. Luznik, "Cyclo-Phosphamide Resets Dendritic Cell Homeostasis and Enhances Antitumor Immunity through Effects that Extend Beyond Regulatory T Cell Elimination," Cancer Immunology Immunotherapy, Vol. 59, No. 1, 2010, pp. 137 148. doi:10.1007/s00262-009-0734-3

[28] P. Liu, J. Jaffar, I. Hellstrom and K. Hellstrom, “Administration of Cyclophosphamide Changes the Immune Profile of Tumor-Bearing Mice," Journal of Immunotherapy, 
Vol. 33, No. 1, 2010, pp. 53-59. doi:10.1097/CJI.0b013e3181b56af4

[29] R. Goforth, A. Salem, X. Zhu, S. Miles, X. Zhang, J. Lee and A. Sandler, "Immune Stimulatory Antigen Loaded Particles Combined with Depletion of Regulatory T-Cells Induce Potent Tumor Specific Immunity in a Mouse Model of Melanoma," Cancer Immunology Immunotherapy, Vol. 58, No. 4, 2009, pp. 517-530. doi:10.1007/s00262-008-0574-6

[30] N. Wang and X. Wu, "Synthesis, Characterization, Bio- degradation, and Drug Delivery Application of Biodegradable Lactic/Glycolic Acid Oligomers: Part II. Biodegradation and Drug Delivery Application," Journal of Biomaterials Science, Polymer Edition, Vol. 9, 1997, pp. 75-87.

[31] K. Khazale and H. von Boehmer, "The Impact of CD4+CD25+ Treg on Tumor Specific CD8+ T Cell Cytotoxicity and Cancer," Seminars in Cancer Biology, Vol. 16, No. 2, 2006, pp. 124-136. 